\title{
Atomic structure of a metal-supported two-dimensional germania film
}

\author{
Adrián Leandro Lewandowski, ${ }^{1}$ Philomena Schlexer, ${ }^{2}$ Christin Büchner, ${ }^{3}$ Earl M. Davis, ${ }^{1}$ Hannah Burrall, ${ }^{4}$ \\ Kristen M. Burson, ${ }^{4}$ Wolf-Dieter Schneider, ${ }^{1}$ Markus Heyde, ${ }^{1,}{ }^{*}$ Gianfranco Pacchioni, ${ }^{2}$ and Hans-Joachim Freund ${ }^{1}$ \\ ${ }^{1}$ Fritz-Haber-Institut der Max-Planck-Gesellschaft, Faradayweg 4-6, 14195 Berlin, Germany \\ ${ }^{2}$ Department of Materials Science, Università di Milano-Bicocca, Via R. Cozzi, 55, Milan, Italy \\ ${ }^{3}$ Lawrence Berkeley National Laboratory, 1 Cyclotron Road, Mailstop 2R0300, Berkeley, California 94720, USA \\ ${ }^{4}$ Taylor Science Center, Hamilton College, 198 College Hill Road, Clinton, New York 13323, USA
}

(Received 25 August 2017; revised manuscript received 6 February 2018; published 6 March 2018)

\begin{abstract}
The growth and microscopic characterization of two-dimensional germania films is presented. Germanium oxide monolayer films were grown on $\mathrm{Ru}(0001)$ by physical vapor deposition and subsequent annealing in oxygen. We obtain a comprehensive image of the germania film structure by combining intensity-voltage low-energy electron diffraction (I/V-LEED) and ab initio density functional theory (DFT) analysis with atomic-resolution scanning tunneling microscopy (STM) imaging. For benchmarking purposes, the bare $\mathrm{Ru}(0001)$ substrate and the $(2 \times 2) 3 \mathrm{O}$ covered $\mathrm{Ru}(0001)$ were analyzed with I/V-LEED with respect to previous reports. STM topographic images of the germania film reveal a hexagonal network where the oxygen and germanium atom positions appear in different imaging contrasts. For quantitative LEED, the best agreement has been achieved with DFT structures where the germanium atoms are located preferentially on the top and fcc hollow sites of the $\mathrm{Ru}(0001)$ substrate. Moreover, in these atomically flat germania films, local site geometries, i.e., tetrahedral building blocks, ring structures, and domain boundaries, have been identified, indicating possible pathways towards two-dimensional amorphous networks.
\end{abstract}

DOI: 10.1103/PhysRevB.97.115406

\section{INTRODUCTION}

Using thin oxide films represents an elegant way to apply electron-based analytical methods to unravel the atomic surface structure of insulating materials [1]. Recently, we have investigated silica surfaces and have demonstrated that both crystalline as well as vitreous two-dimensional (2D) films can be prepared and resolved at the atomic level using scanning probe techniques [2]. Interestingly, our findings on vitreous silica films verify the random network hypothesis by Zachariasen dated 1932, which is conceptionally applied to understand three-dimensional amorphous networks [3]. For vitreous bulk materials, direct experimental information on atomic coordinates is not available [4]. There is a discussion in the literature that germania in comparison to silica will have different properties, in particular with respect to the formation of vitreous, i.e., glassy, phases [5-7]. Also, doping of germanium into silica or a mixed phase of both might lead to promising materials in a number of applications [8-11]. Given the detailed information derived from thin-film studies for silica, it is likely that one will be able to reveal the network structures for germania and germania-silica mixtures at the atomic level.

\footnotetext{
*heyde@fhi-berlin.mpg.de

Published by the American Physical Society under the terms of the Creative Commons Attribution 4.0 International license. Further distribution of this work must maintain attribution to the author(s) and the published article's title, journal citation, and DOI.
}

In the present study, a thin-film strategy is employed for the identification of local site geometries, tetrahedral building blocks, and larger building units, i.e., rings. Preparation of and experiments on ultrathin germania films grown on $\mathrm{Ru}(0001)$ were performed under ultrahigh vacuum (UHV) conditions. The film consists of a crystalline single layer of corner-sharing $\mathrm{GeO}_{4}$ tetrahedra forming a continuous network structure. Atomically resolved images were obtained with scanning tunneling microscopy (STM). Different imaging contrasts reveal the atomic positions of germanium and oxygen atoms, which are arranged in a hexagonal lattice. Intensity-voltage low-energy electron diffraction (I/V-LEED) studies together with $a b$ initio density functional theory (DFT) models provide a consistent view of the germania film structure. First characterization of possible ring structures, ring configurations, and domain boundaries has been made.

\section{EXPERIMENTAL DETAILS}

The experiments were carried out in an UHV vacuum chamber $\left(2 \times 10^{-10} \mathrm{mbar}\right)$ equipped with a Beetle-type STM, LEED, and standard facilities for sample preparation. Details of the experimental setup can be found in Ref. [12].

Ultrathin germania films were grown on a clean $\mathrm{Ru}(0001)$ single crystal by first establishing a $(2 \times 2)-3 \mathrm{O}$ overlayer and subsequently depositing germanium (Aldrich, purity $\geqslant 99.999 \%$ ) by electron-beam evaporation at room temperature in $2 \times 10^{-6} \mathrm{mbar}$ of oxygen pressure. After Ge deposition, annealing the sample for $10 \mathrm{~min}$ at $850 \mathrm{~K}$ in the same oxygen environment and cooling down by simply switching off the heater produces an atomically flat film. 
The STM measurements were obtained at room temperature. The details and individual imaging parameters are given in the corresponding figure captions.

All I/V-LEED measurements were recorded at room temperature and normal incidence. Fine-tuned alignment between the sample and the electron beam was achieved by generating a uniform magnetic field with a Helmholtz coil. A LABVIEW script sets the beam energy of the LEED controller and the current of the Helmholtz coils via a digital-analog converter, and takes images from a Grundig FA87 video camera for each energy with a video grabber unit (see Supplemental Material Fig. S1 for a diagram of the setup [13]). For the analysis, symmetry-equivalent spots, showing the same intensity maxima and minima in their I/V-LEED curves, were considered together and their intensities were averaged. For the germania monolayer, a range of $60-540 \mathrm{eV}$ was used for the electron kinetic energy, with steps of $1 \mathrm{eV}$. The intensity of 42 diffraction spots was recorded, giving a total energy range of $2450 \mathrm{eV}$. The intensity-voltage curves were normalized by the electron current reaching the sample and smoothed to remove experimental noise. Experimental curves were reproducible for films having a coverage of $1.00 \pm 0.05 \mathrm{ML}$. A series of consecutive kinetic energy ramps at a fixed sample location does not show changes in the I/V-LEED curves, indicating negligible damage of the film due to electron bombardment. In addition, this has been verified by STM images before and after LEED experiments. The films show flat terraces without apparent changes when investigated over several days under UHV conditions with STM and LEED.

Theoretical I/V-LEED curves were calculated using a modified version of the Symmetrized Automated Tensor LEED (SATLEED) package of Barbieri and van Hove that also includes a program for the calculation of relativistic phase shifts [14]. Experimental and theoretical I/V-LEED curves were quantitatively compared using the Pendry reliability factor $\mathrm{R}_{P}$ [15]. The Debye temperatures, muffin-tin radii (for oxygen, ruthenium, and germanium), and the inner potential were optimized in pursuit of lower $\mathrm{R}$ factors. A maximum angular momentum 1_max $=10$ was used. The initial structural parameters were based on information coming from the structure optimization of DFT calculations. Periodic, spin-polarized DFT calculations including dispersion forces were performed using the Vienna Ab Initio Simulation Package (VASP) [16-19]. We used the Perdew-Burke-Ernzerhof (PBE) exchange-correlation functional $[20,21]$ and the projector augmented wave (PAW) method for electron-ion interactions. More details are given in the Supplemental Material [13]. To model the Ru-supported germania monolayer, we started from the well-known atomic structure of the silica monolayer [22].

\section{RESULTS AND DISCUSSION}

Figure 1(a) presents a $200.0 \mathrm{~nm} \times 84.0 \mathrm{~nm}$ STM image which shows nearly complete coverage of the atomically flat germania film on three $\mathrm{Ru}(0001)$ terraces. Different types of grain boundaries can also be observed in the film. Structural details of such domain boundaries have been resolved in Fig. 1(b).

In a series of STM images shown in Figs. 1(c) and 1(d) we observed different contrasts on the germania monolayer film.
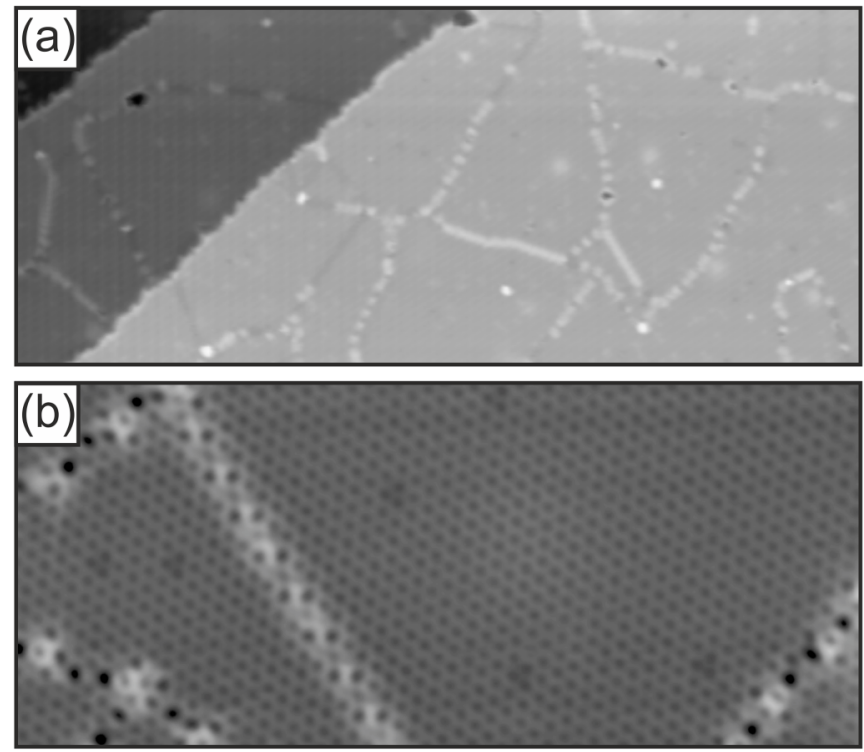

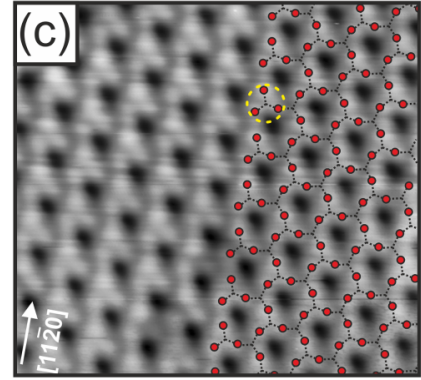

- 0

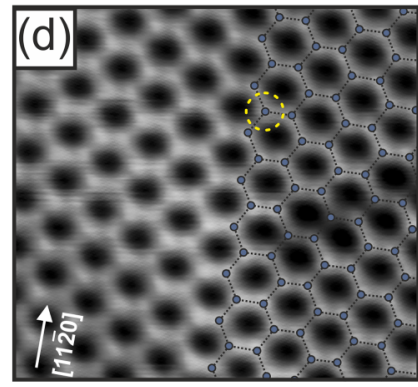

- $\mathrm{Ge}$
FIG. 1. Overview and atomically resolved STM images of the germania monolayer film supported on $\mathrm{Ru}(0001)$. (a) Almost closed germania film imaged across substrate step edges, $200.0 \mathrm{~nm} \times$ $84.0 \mathrm{~nm}, I_{T}=600 \mathrm{pA}, V_{S}=3.0 \mathrm{~V}$. (b) Characteristic domain boundaries in the germania film resolved, $30.0 \mathrm{~nm} \times 13.0 \mathrm{~nm}$, $I_{T}=600 \mathrm{pA}, V_{S}=2.5 \mathrm{~V}$. Different atomic contrasts are observed in (c) and (d). The top view of the tetrahedral building unit derived from the imaging contrast has been indicated by a yellow dashed circle in each figure. The contrast in (c) can be assigned to $\mathrm{O}$ positions marked by red dots, $4.1 \mathrm{~nm} \times 4.1 \mathrm{~nm}, I_{T}=300 \mathrm{pA}, V_{S}=0.2 \mathrm{~V}$. While (d) has the same scan size as (c), the STM image contrast corresponds to Ge positions superimposed with blue dots, $4.1 \mathrm{~nm} \times 4.1 \mathrm{~nm}$, $I_{T}=500 \mathrm{pA}, V_{S}=2.0 \mathrm{~V}$.

Both STM images have a size of $4.1 \mathrm{~nm} \times 4.1 \mathrm{~nm}$. The observed structures point towards different chemical sensitivity. Note that Figs. 1(c) and 1(d) were obtained on different positions of the surface, which are assumed to be structurally equivalent. The STM images are superimposed with red and blue markers to show the positions of oxygen and germanium atoms, respectively. In Fig. 1(c) the motif of three protrusions in a triangle is in agreement with the three $\mathrm{O}$ atoms that are exposed when observing one face of the $\mathrm{GeO}_{4}$ tetrahedron. On the other hand, Fig. 1(d) shows a honeycomblike structure, with protrusions forming hexagons or six-membered rings revealing Ge atomic positions. The resulting network topology is in agreement with equivalent imaging contrasts that have been reported for $\mathrm{O}$ and Si sites in silica films [23].

Depending on the electronic states involved in the tunneling process, STM probes can provide different element sensitive 
contrasts of the surface structures. The calculated partial density of states of the system (see Fig. S7 in the Supplemental Material [13]) shows that oxygen contributes more at lower bias than germanium, which is in line with our contrast assignment in the STM images.

However, simply changing the sample voltage does not immediately cause a change in the image contrast. Therefore the tip termination also seems to play an important role in the formation of the image contrast. It is known for STM and AFM that the composition and the structure of the tip apex can also be responsible for different chemically resolved images [24,25].

In order to clarify the role of each step in the sample preparation, STM and LEED data were acquired throughout the process, for clean $\mathrm{Ru}(0001), \mathrm{Ru}(0001)-(2 \times 2)-3 \mathrm{O}$, and $\mathrm{a}$ monolayer of germania on $\mathrm{Ru}(0001)-(2 \times 2)-30$. The clean $\mathrm{Ru}(0001)$ shows $(1 \times 1)$ reflexes in a hexagonal pattern in the LEED image [see Fig. 2(a)]. The $(1 \times 1)$ unit cell is indicated on the accompanying structural model. The addition of the oxygen overlayer forms a $(2 \times 2)-30$ structure, revealed by a less intense hexagonal spot pattern in Fig. 2(b). The larger $(2 \times 2)$ unit cell is also indicated in Fig. 2(b). Finally, after vapor deposition of $\mathrm{Ge}$ and a high-temperature oxidation step in the same oxygen environment, the germania monolayer preparations exhibit a $(2 \times 2)$ superstructure in LEED, commensurate with the lattice of the $\mathrm{Ru}(0001)$ surface [Fig. 2(c)].

I/V-LEED is utilized in order to experimentally assess the full germania structure and the presence of adsorbed oxygen between film and substrate. Importantly, I/V-LEED is a quantitative technique which can probe structures beyond the topmost surface layer and, therefore, can address structural configurations that are inaccessible with STM alone.

I/V-LEED was measured and calculated for a Ru(0001) single crystal, a $(2 \times 2)-30$ structure on $\mathrm{Ru}(0001)$, and finally the deposited germania film on the oxygen precovered $\mathrm{Ru}(0001)$. The analysis of the first two systems provides a built-in check for the veracity of our setup through comparison with literature. For clean $\mathrm{Ru}(0001)$ and $\mathrm{Ru}(0001)-(2 \times 2)-3 \mathrm{O}$, an overall data set of $1431 \mathrm{eV}$ and $2082 \mathrm{eV}$ was used with $\mathrm{R}_{P}$ of 0.13 and 0.18 , respectively, both lower than those reported in literature $[26,27]$. Refer to the Supplemental Material for the I/V-LEED curves [13].

As DFT calculations of the germania bilayer suggest a distorted structure [28], we also tested a distorted geometry for the monolayer shown in Fig. 2(c), where the tetrahedral building blocks are slightly twisted against each other. For the germania films, nine different DFT models were used as reference structures in the calculations, mainly characterized by distorted and nondistorted structures, additional surface oxygen, and different registries between film and substrate, which we denote as hcp, fcc, and top. These terms refer to the position of the germania honeycomb centers with respect to the Ru lattice. For the I/V-LEED curve calculations, atomic coordinates derived from DFT were further optimized using the Tensor LEED approximation.

The DFT model in Fig. 2(c) agrees with both atomic STM contrast observations shown in Figs. 1(c) and 1(d). The atomic model is formed from the superposition of the oxygen and germanium contrasts. The germania monolayer is strongly bound to the $\mathrm{Ru}$ surface, just as the silica monolayer is [29].
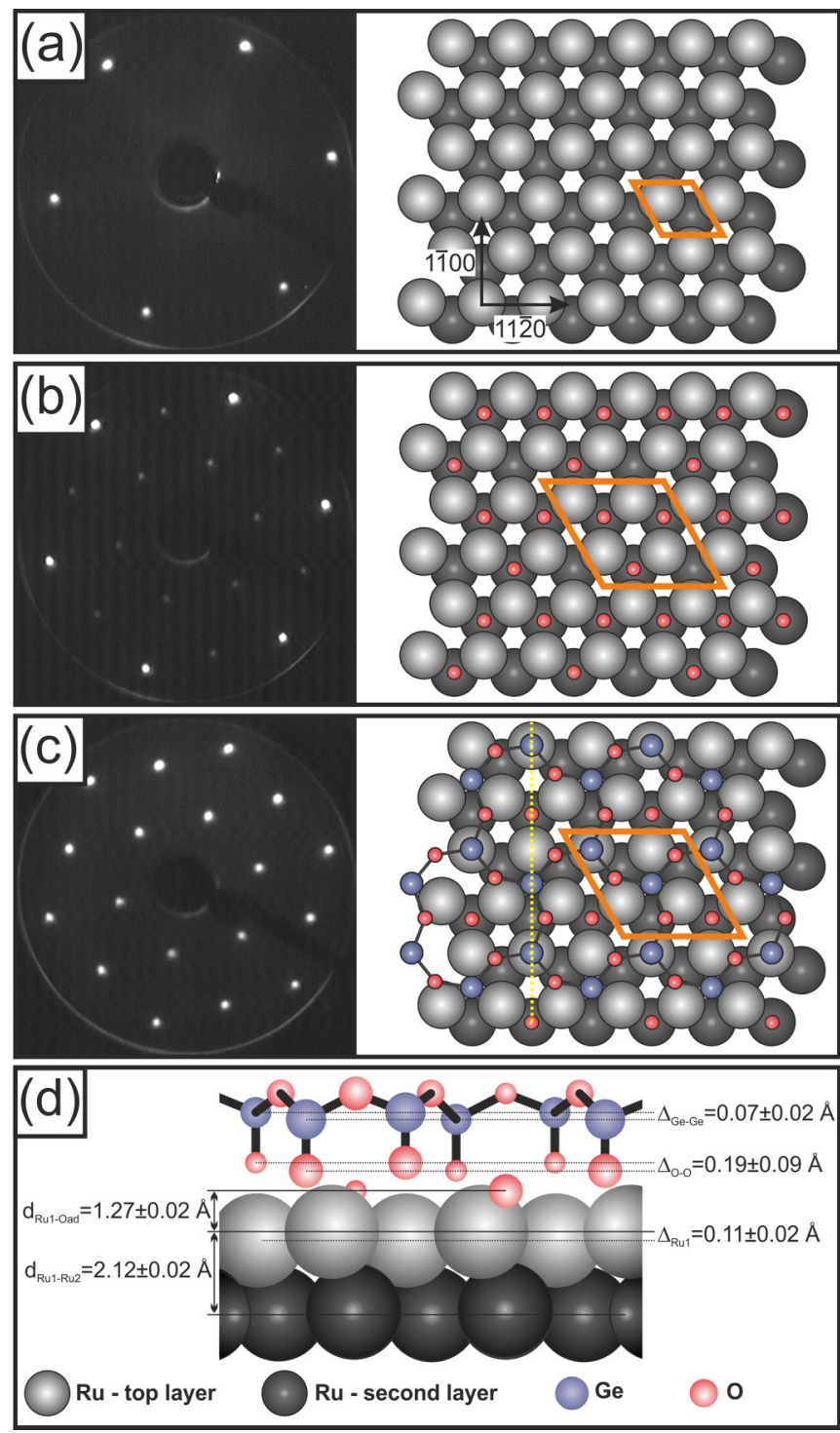

FIG. 2. Preparation steps of germania films assessed by LEED and DFT. The LEED images were taken at $E_{k i n}=60 \mathrm{eV}$ and are given together with the corresponding structural models from DFT and I/V-LEED analysis. For each model the unit cell is shown in orange. (a) clean $\mathrm{Ru}(0001)$ crystal, (b) $\mathrm{Ru}(0001)-(2 \times 2)-3 \mathrm{O}$, (c) germania film in a hcp configuration with oxygen on $\mathrm{Ru}(0001)$, and (d) side view of the model in (c) at the plane indicated by the yellow dashed line. The atomic coordinates are the result of the optimization process of I/V-LEED. Drawing is not to scale and height variations are exaggerated for better visualization. At the bottom a legend of the atoms involved is provided.

From DFT we also found the hcp configuration of the germania film to be the most stable one for the pristine $\mathrm{Ru}$ surface, as well as for the Ru surface with additional surface oxygen. In both cases, the fcc and top registries are less stable than the hcp. (See Table S1 and Fig. S5 in the Supplemental Material [13].)

The presence of the oxygen adatoms shown in Fig. 2(c) slightly decreases the adhesion energy of the distorted germania monolayer with respect to the adsorbate-free system. (See Table S1 in the Supplemental Material [13].) The presence of 


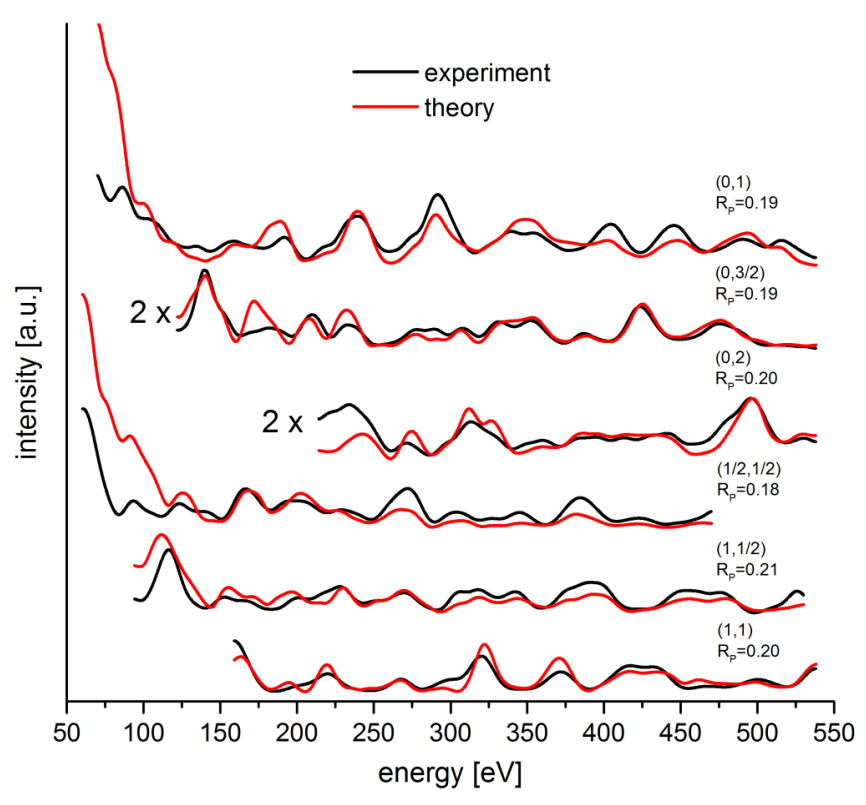

FIG. 3. Comparison between theoretical and experimental I/V-LEED curves for germania on $\mathrm{Ru}(0001)$. The chosen reciprocal lattice spots are indicated by their Miller indices. The distorted germania monolayer at the hcp site with additionally adsorbed atomic oxygen is plotted and provides an $\mathrm{R}_{P}$ of 0.19 .

adsorbed oxygen atoms in the middle of every six-membered ring on hep hollow sites is consistent with the preferred adsorption site for oxygen adlayers on $\mathrm{Ru}(0001)$ [27,30]. An analog structure has been derived for a silica monolayer on $\mathrm{Ru}(0001)$ [31].

In the I/V-LEED analysis the comparison between the calculated and experimental curves for the distorted hcp structure with adsorbed oxygen is shown in Fig. 3, while other models and corresponding $\mathrm{R}_{P}$ are provided in the Supplement [13]. The fcc and top models yield $\mathrm{R}_{P}>0.40$. The optimum $\mathrm{R}$ factors show a clear preference for the hcp distorted structures. In particular, the one with adsorbed atomic oxygen [see Fig. 2(c)] shows the best $\mathrm{R}_{P}=0.19$. This germania monolayer model obtained from the Tensor LEED optimization process gives the lowest $\mathrm{R}_{P}$ and is described next. The structure is crystalline and consists of tetrahedral $\mathrm{GeO}_{4}$ building blocks which are bound to the substrate through oxygen atoms to top and fcc hollow sites of the $\mathrm{Ru}(0001)$. Adjacent tetrahedra share an oxygen atom and are rotated by $30^{\circ}$ around an axis perpendicular to the surface. Refer to the schemes of Figs. 2(c) and 2(d) for the top and side view of this system. In 2D, the network forms hexagons of six Ge atoms interconnected with six $\mathrm{O}$ atoms, simply called six-membered rings.

Figure 2(d) specifies some layer distances and height variations within the layers. In order to estimate their error bars, the atoms involved were simultaneously displaced by the same amount from their minimum positions along the vertical direction. From the graph of the displacement versus the $\mathrm{R}$ factor the curvature was extracted and the Pendry approach was used [15]. The error bars of individual atoms along Cartesian coordinate directions are shown in the Supplemental Material [13]. The structural model presents a vertical rumpling due to two different binding schemes. Ge and $\mathrm{O}$ atoms on top positions occur higher than the ones on fcc hollow sites, as shown in $2(\mathrm{~d})$ for $\Delta_{\mathrm{Ge}-\mathrm{Ge}}$ and $\Delta_{\mathrm{O}-\mathrm{O}}$. Ge-O bonds perpendicular to the surface on fcc hollow sites are significantly longer than the ones on top positions: $1.84 \pm 0.03 \AA$ versus $1.73 \pm 0.03 \AA$. However, the $\mathrm{O}$ atoms in the topmost layer are all at the same height. To compensate for that, different $\mathrm{O}-\mathrm{Ge}-\mathrm{O}$ angles occur in a range of $103^{\circ}$ to $114^{\circ}$. The Ge-O-Ge bond forms an angle of $129 \pm 2^{\circ}$. The oxygen atoms on hcp hollow sites are adsorbed at $1.27 \pm 0.02 \AA$ with respect to the first ruthenium layer, further away than the calculated value for $\mathrm{Ru}(0001)$ $(2 \times 2)-3 \mathrm{O}$ of $1.20 \pm 0.02 \AA$. The contraction of the first $\mathrm{Ru}$ layer yields comparable results with the calculations on bare ruthenium. Those $\mathrm{Ru}$ atoms involved in the $\mathrm{Ru}-\mathrm{O}-\mathrm{Ge}$ bond [dashed line in Fig. 2(d)] are found considerably lower than the neighboring $\mathrm{Ru}$ atoms (solid line). The $\mathrm{Ru}-\mathrm{O}$ bond therein has a length of $1.68 \pm 0.02 \AA$. More details of the structure of the film and the reconstruction of the metallic substrate are given in the Supplemental Material [13]. Both the bond lengths and the angles that are involved in this film network structure are within the measured range for bulk amorphous $\mathrm{GeO}_{2}$ using neutron diffraction, $\mathrm{x}$-ray diffraction, and anomalous $\mathrm{x}$-ray scattering $[7,32]$. In addition to this agreement with bulk germania structural parameters, the results from the structure optimization process of I/V-LEED are in line with the two different atomic contrasts observed by STM.

Besides the ideal hexagonal lattice, other ring sizes are also present in germania films. In our study, a variation in ring size is most commonly observed in the form of domain boundaries and connection points between boundaries, as already visible in Figs. 1(a) and 1(b).

It is thought that domain boundaries are formed to compensate for translations between crystalline domains as they grow and to maintain the atomic arrangements between the monolayer and substrate. According to the DFT results, it is energetically favorable for the crystalline germania lattice to be positioned in the hcp configuration with respect to the $\mathrm{Ru}(0001)$ substrate. I/V-LEED results, coupled with observations of sizable domains in STM [Figs. 1(a) and 1(b)], suggest that the atomic arrangement of the crystalline domains relative to the $\mathrm{Ru}(0001)$ substrate on either side of the boundary exhibit the energetically favorable orientation. This is further confirmed by models of the relationship between the germania and $\mathrm{Ru}(0001)$ substrate (see Supplemental Material [13]).

Detailed examples of antiphase domain boundaries in germania monolayers on $\mathrm{Ru}(0001)$ are shown in Fig. 4, and are classified according to their ring distribution.

STM images in Figs. 4(a) and 4(b) show the highly symmetric 48 boundary, which alternates linearly between four- and eight-membered rings. The same arrangement has been reported for domain boundary structures present in silica monolayer films on $\operatorname{Mo}(112)$ [33,34], silica bilayer films on $\mathrm{Ru}(0001)$ [35], and aluminosilicate ultrathin films on $\mathrm{Ru}(0001)$ [36].

The 57756 boundary in Figs. 4(c) and 4(d) is a combination of Stone-Wales defects [37] arranged linearly that are separated by a six-membered ring in the germania monolayer film structure. Comparable 5577 structural motifs have been found in domain boundaries as well as in isolated rectangular loop defects in silica monolayer films on $\mathrm{Ru}(0001)$ with a slightly different arrangement [31,35,38]. 

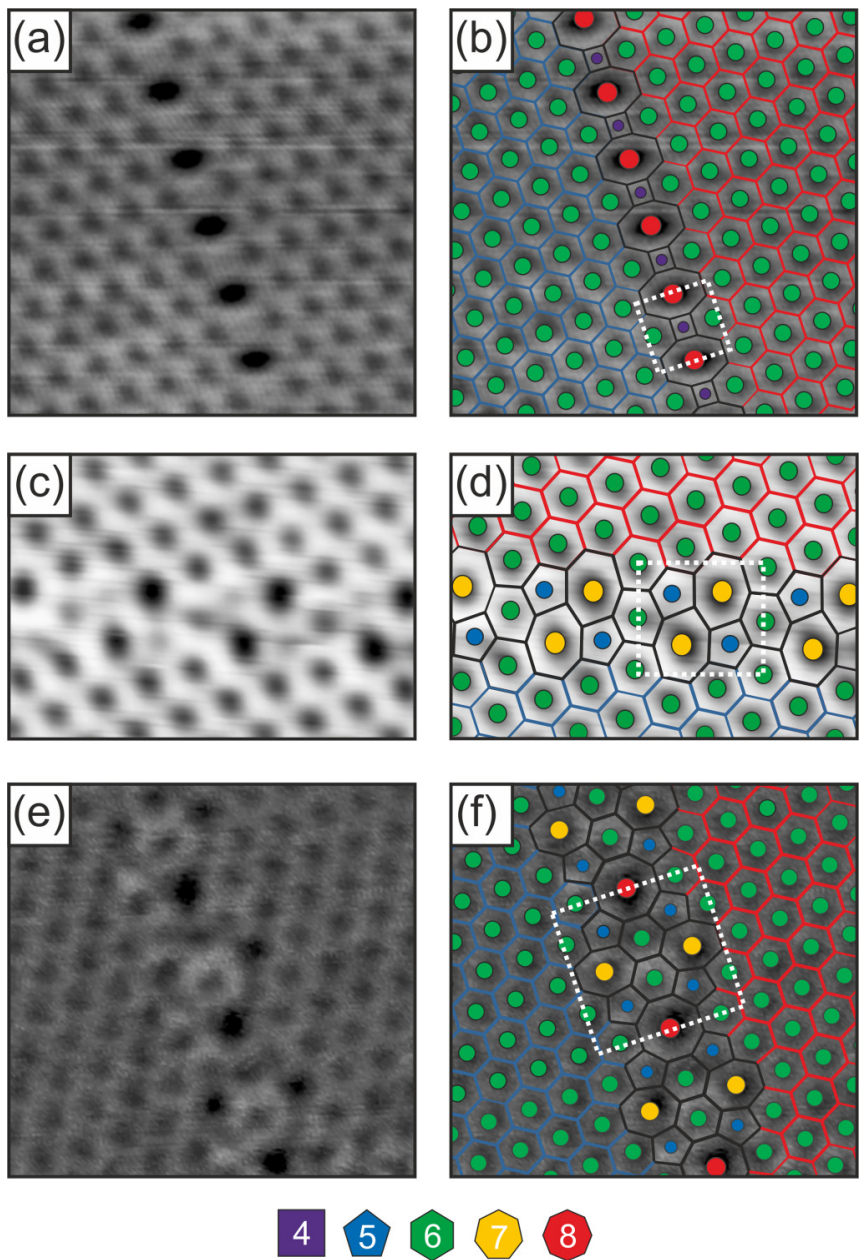

FIG. 4. Three different boundary structures commonly found in germania monolayer films. For each STM image, the size of the rings is highlighted with color-coded circles in the right-hand column and white dotted squares show the unit cells of the boundaries. $(a, b) 84$ boundary, scan range $5.5 \mathrm{~nm} \times 5.7 \mathrm{~nm}, I_{T}=4.5 \mathrm{nA}, V_{S}=2.4 \mathrm{~V}$. (c, d) 57756 boundary $5.6 \mathrm{~nm} \times 4.9 \mathrm{~nm}, I_{T}=500 \mathrm{pA}, V_{S}=2.5 \mathrm{~V}$. (e, f) Complex boundary, formed by five-, six-, seven-, and eight-membered rings, $6.3 \mathrm{~nm} \times 6.1 \mathrm{~nm}, I_{T}=4 \mathrm{nA}, V_{S}=2.4 \mathrm{~V}$.

The most complex boundary structure for the germania monolayer film is the one shown in Figs. 4(e) and 4(f). Here, we have observed a formation with five-, six-, seven-, and eight-membered rings, which is complex, but also ordered and periodic. Such a boundary structure so far has not been observed in silica film systems. This domain boundary exhibits in its ring arrangement almost zeolitelike features while maintaining its 2D flat film structure. This enrichment in ring size distribution opens up a possible path for 2D glass configurations.

So far the observed germania monolayer film network seems to be fairly comparable to silica film structures. However, our results from STM, I/V-LEED, and DFT for the hexagonal germania monolayers predict a slight variation in the angular arrangement of the tetrahedral building units and a more coupled interaction of the film system with the Ru metal substrate. Furthermore, additional domain boundaries with a broad ring size distribution have been found.

\section{CONCLUSIONS}

The present work addresses the successful preparation of germania monolayer films on $\mathrm{Ru}(0001)$ and their structural characterization using STM, DFT, and I/V-LEED. The atomic structure consists predominantly of hexagons with $\mathrm{Ge}$ atoms in the corners sitting on top and fcc hollow sites of $\mathrm{Ru}(0001)$. The germania monolayer film has an areal density of germanium of $0.95 \mathrm{mg} / \mathrm{m}^{2}$. The corner-sharing $\mathrm{GeO}_{4}$ building blocks are linked to the substrate via $\mathrm{O}$ atoms. Oxygen atoms adsorb on hcp hollow sites in the middle of each six-membered ring. By combining I/V-LEED, DFT, and STM, a full picture of the crystalline germania monolayer structure has been obtained. Line defects in the film introduce ring sizes that vary from the ideal hexagonal lattice. These findings point to the possibility to create a $2 \mathrm{D}$ glass of germania or of mixtures of germania and silica which would be new members of the family of 2D glass-forming oxide materials.

\section{ACKNOWLEDGMENTS}

The authors would like to thank S. Elliott for helpful discussions and Helmut Kuhlenbeck for the help setting up the I/VLEED system. K.M.B. gratefully acknowledges the support of the Alexander von Humboldt Foundation. C.B. is grateful to the CRC 1109, funded by the Deutsche Forschungsgemeinschaft, for financial support. P.S. and G.P. gratefully acknowledge support from the European Marie Curie project CATSENSE (Grant Agreement No. 607417). This project has received funding from the European Research Council (ERC) under the European Union's Horizon 2020 Research and Innovation Program (Grant Agreement No. 669179).
[1] L. Giordano and G. Pacchioni, Acc. Chem. Res. 44, 1244 (2011).

[2] L. Lichtenstein, C. Büchner, B. Yang, S. Shaikhutdinov, M. Heyde, M. Sierka, R. Włodarczyk, J. Sauer, and H.-J. Freund, Angew. Chem. Int. Ed. 51, 404 (2012).

[3] W. H. Zachariasen, J. Am. Chem. Soc. 54, 3841 (1932).

[4] A. C. Wright, J. Non-Cryst. Solids 179, 84 (1994).

[5] P. S. Salmon, A. C. Barnes, R. A. Martin, and G. J. Cuello, J. Phys.: Condens. Matter 19, 415110 (2007).
[6] M. Micoulaut, L. Cormier, and G. S. Henderson, J. Phys.: Condens. Matter 18, R753 (2006).

[7] D. L. Price, M.-L. Saboungi, and A. C. Barnes, Phys. Rev. Lett. 81, 3207 (1998).

[8] G. S. Henderson, D. R. Neuville, B. Cochain, and L. Cormier, J. Non-Cryst. Solids 355, 468 (2009).

[9] O. Majerus, L. Cormier, D. R. Neuville, L. Galoisy, and G. Calas, J. Non-Cryst. Solids 354, 2004 (2008). 
[10] E. M. Dianov and V. M. Mashinsky, J. Lightwave Technol. 23, 3500 (2005).

[11] C. M. Zicovich-Wilson and A. Corma, J. Phys. Chem. B 104, 4134 (2000).

[12] N. Nilius, A. Cörper, G. Bozdech, N. Ernst, and H.-J. Freund, Prog. Surf. Sci. 67, 99 (2001).

[13] See Supplemental Material at http://link.aps.org/supplemental/ 10.1103/PhysRevB.97.115406 for sample preparation, LEED structure determination, DFT calculations, domain boundary structures, and germania coordinates from I/V-LEED and DFT.

[14] A. Barbieri and M. van Hove (private communication), http://www.icts.hkbu.edu.hk/vanhove/.

[15] J. B. Pendry, J. Phys. C: Solid State Phys. 13, 937 (1980).

[16] G. Kresse and J. Hafner, Phys. Rev. B 47, 558(R) (1993).

[17] G. Kresse and J. Hafner, Phys. Rev. B 49, 14251 (1994).

[18] G. Kresse and J. Furthmüller, Comput. Mater. Sci. 6, 15 (1996).

[19] G. Kresse and J. Furthmüller, Phys. Rev. B 54, 11169 (1996).

[20] J. P. Perdew, K. Burke, and M. Ernzerhof, Phys. Rev. Lett. 77, 3865 (1996).

[21] J. P. Perdew, K. Burke, and M. Ernzerhof, Phys. Rev. Lett. 78, 1396 (1997).

[22] D. Löffler, J. J. Uhlrich, M. Baron, B. Yang, X. Yu, L. Lichtenstein, L. Heinke, C. Büchner, M. Heyde, S. K. Shaikhutdinov, H.-J. Freund, R. Włodarczyk, M. Sierka, and J. Sauer, Phys. Rev. Lett. 105, 146104 (2010).

[23] L. Lichtenstein, M. Heyde, and H.-J. Freund, J. Phys. Chem. C. 116, 20426 (2012).

[24] M. Schmid, H. Stadler, and P. Varga, Phys. Rev. Lett. 70, 1441 (1993).
[25] Y. Sugimoto, P. Pou, M. Abe, P. Jelinek, R. Perez, S. Morita, and O. Custance, Nature (London) 446, 64 (2007).

[26] G. Michalk, W. Moritz, H. Pfnür, and D. Menzel, Surf. Sci. 129, 92 (1983).

[27] M. Gsell, M. Stichler, P. Jakob, and D. Menzel, Isr. J. Chem. 38, 339 (1998).

[28] A. Malashevich, S. Ismail-Beigi, and E. I. Altman, J. Phys. Chem. C 120, 26770 (2016).

[29] L. Lichtenstein, M. Heyde, S. Ulrich, N. Nilius, and H.-J. Freund, J. Phys.: Condens. Matter 24, 354010 (2012).

[30] C. Stampfl, S. Schwegmann, H. Over, M. Scheffler, and G. Ertl, Phys. Rev. Lett. 77, 3371 (1996)

[31] S. Mathur, S. Vlaic, E. Machado-Charry, A.-D. Vu, V. Guisset, P. David, E. Hadji, P. Pochet, and J. Coraux, Phys. Rev. B 92, 161410(R) (2015).

[32] J. E. Desa, A. C. Wright, and R. N. Sinclair, J. Non-Cryst. Solids 99, 276 (1988).

[33] J. Weissenrieder, S. Kaya, J.-L. Lu, H.-J. Gao, S. Shaikhutdinov, H.-J. Freund, M. Sierka, T. K. Todorova, and J. Sauer, Phys. Rev. Lett. 95, 076103 (2005).

[34] S. Ulrich, N. Nilius, H.-J. Freund, U. Martinez, L. Giordano, and G. Pacchioni, Chem. Phys. Chem. 9, 1367 (2008).

[35] K. M. Burson, C. Büchner, M. Heyde, and H.-J. Freund, J. Phys.: Condens. Matter 29, 035002 (2017).

[36] J. A. Boscoboinik, X. Yu, B. Yang, S. Shaikhutdinov, and H.-J. Freund, Microporous Mesoporous Mater. 165, 158 (2013).

[37] A. Stone and D. Wales, Chem. Phys. Lett. 128, 501 (1986).

[38] B. Yang, J. A. Boscoboinik, X. Yu, S. Shaikhutdinov, and H.-J. Freund, Nano Lett. 13, 4422 (2013). 